\title{
Randomised Study of early Continuous positive airways pressure in Acute Respiratory Failure in children with impaired immunity (SCARF) ISRCTN82853500
}

\author{
Mark J Peters ${ }^{1,2}$, Rachel Agbeko ${ }^{3,4}$, Peter Davis ${ }^{5}$, Nigel Klein ${ }^{6}$, Zohra Zenasi ${ }^{7}$, Amy Jones², \\ Christine Mackerness ${ }^{3}$, Susan George ${ }^{5}$, Paul Veys ${ }^{8}$, Samiran Ray ${ }^{1,2}$, Paul R Mouncey ${ }^{7}$, David A. \\ Harrison ${ }^{7}$ and Kathy Rowan ${ }^{7}$ on behalf of the SCARF study investigators and the Paediatric \\ Intensive Care Society Study Group (PICS-SG)
}

1.Respiratory Critical Care and Anaesthesia Unit, UCL Great Ormond Street Institute of Child Health, London. WC1N 1EH

2. Paediatric and Neonatal Intensive Care Units, Great Ormond Street Hospital for Children NHS Trust, London. WC1N 3JH

3. Paediatric Intensive Care Unit, Great North Children's Hospital, Victoria Wing, Royal Victoria Infirmary, Newcastle upon Tyne NE1 4LP

4. Institute of Cellular Medicine, Newcastle University, Framlington Place, Newcastle upon Tyne NE2 4HH

5. Paediatric Intensive Care Unit, Bristol Royal Hospital for Children, Upper Maudlin St, Bristol BS2 8BJ

6. Infectious Disease and Microbiology Unit, UCL Great Ormond Street Institute of Child Health, London. WC1N $1 \mathrm{EH}$

7. Clinical Trials Unit, Intensive Care National Audit \& Research Centre, Napier House 24 High Holborn, London, WC1V 6AZ

8. Department of Immunology and Bone Marrow Transplantation, Great Ormond Street Hospital, London, United Kingdom;

\section{Corresponding author}

Professor Mark J Peters

Critical Care Group - UCL Great Ormond Street Institute of Child Health, London. WC1N 1EH mark.peters@ucl.ac.uk

T +442078138213

F +442078138206

Keywords, PICU respiratory failure, ARDS, Immunodeficiency CPAP, Non-invasive ventilation Word count $3135 \quad$ Abstract 251 
Copyright form disclosure: Dr. Peters and Ms. George received support for article research from the Great Ormond Street Hospital (GOSH) Children's Charity. Registered Charity No. 235825. Dr. Peters disclosed that the study was supported by the National Institute for Health Research (NIHR) GOSH Biomedical Research Centre, and he received funding from Faron Pharmaceutics and Therakind. Dr. Agbeko's institution received funding from Great Ormond Street Charity, Academic Health Science Network North East North Cumbria, and National Confidential Enquiry into Patient Outcome and Death; she received funding from the NIHR; and she received other support from Children's Research Institute, Washington, D.C. Dr. Davis received support for article research from GOSH Research funds for research nursing time to support study. Dr. Klein's institution received funding from the NIHR, and he received support for article research from the NIHR. Ms. George's institution received funding from ICNARC (intensive Care National Audit \& Research Centre) on behalf of GOSH Children's Charity. Dr. Veys received funding from EUSA and Novartis. Dr. Ray and Mr. Mouncey's institutions received funding from the Department of Health's NIHR Biomedical Research Centre's funding scheme. Mr. Mouncey received support for article research from GOSH Children's Charity. Drs. Harrison and Rowan's institution received funding from GOSH Children's Charity. The remaining authors have disclosed that they do not have any potential conflicts of interest. 


\section{Summary \\ Background}

Previous trials in adults with impaired immunity and respiratory failure suggest that early noninvasive ventilation avoids endotracheal intubation and improves survival. No randomised clinical trials have addressed this question in children.

\section{Methods}

We undertook an open, parallel-group randomised trial of children with impaired immunity and acute respiratory failure defined as: tachypnoea ( $>90^{\text {th }}$ centile); a new requirement for supplemental oxygen; and new chest $x$-ray infiltrates. Children were randomly assigned to early PICU admission for continuous positive airways pressure (early CPAP) or to standard care (standard care). The primary outcome was endotracheal intubation by 30 days.

\section{Findings}

114 children met inclusion criteria of whom 42 were randomised between January 2013 and January 2016. There was no significant difference in endotracheal intubation by 30 days with early CPAP (10/21, 48\%) compared with standard care (5/21, 24\%), odds ratio (OR) 2.9 (0.8-10.9) $p=0.11$. However, 30-day mortality was significantly higher with early CPAP $(7 / 21,33 \%)$ compared to standard care $(1 / 21,5 \%)$ OR $10.0(1.1-90.6) p=0.041$. Mortality at 90 days was: early CPAP $(11 / 21,52 \%)$ versus standard care $(4 / 21,19 \%)$, OR $4.7(1.2-18.6) p=0.029$ while mortality at one year was similar early CPAP (13/21, 61.9\%) versus standard care (9/21, 42.7\%), OR 2.2 (0.6-7.4), $p=0.22$. There were two serious adverse events: early CPAP (pneumothorax) and standard care (haemothorax).

\section{Interpretation}

This study provided no evidence to support early PICU admission for CPAP in children with acute respiratory failure and impaired immunity. There was a trend towards increased endotracheal intubation and a higher early mortality in the early CPAP group.

251 words.

\section{Funding}

This study was funded by Great Ormond Street Hospital Children's Charity. Registered Charity No. 235825 and supported by the National Institute for Health Research Great Ormond Street Hospital Biomedical Research Centre. The views expressed are those of the authors and not necessarily those of the NHS, the NIHR or the UK Department of Health. 
Declaration of Interests

No conflicts of interest to declare. 


\section{Introduction}

Children with impaired immunity represent an increasing subset of paediatric intensive care unit (PICU) admissions. The wider use of immunosuppressive medications and improved survival of children with congenital and acquired immunodeficiencies means that this trend is likely to continue. Observational data show consistently worse outcomes for these children especially in the context of acute respiratory failure - for example, in paediatric acute respiratory distress syndrome, overall mortality is around $20 \%(1)$ but in the context of impaired immunity this approaches $50 \% .(2,3)$

The association of invasive mechanical ventilation with death in adults with impaired immunity prompted a number of studies to determine if non-invasive ventilation could avoid endotracheal intubation and reduce mortality. Three small randomised clinical trials (RCTs) of adults with acute respiratory failure and impaired immunity suggest that using continuous positive airways pressure (CPAP), via facemask or helmet, before it is required to maintain gas exchange, reduces the risk of death by around $60 \% .(4-6)$

None of these studies, however, included children, a group in whom the risks and benefit may differ. Inferences from the adult trials may not be appropriate to children because of differences in respiratory mechanics, (including functional residual capacity being closer to closing volume), differences in case mix, (both in the causes of respiratory failure and the nature of the impaired immunity) but also in the challenges of administering effective CPAP to young children. A further challenge was a lack of data on the incidence and outcome of acute respiratory failure in children with impaired immunity who do not progress to intensive care admission.

We performed a pragmatic RCT to test the hypotheses that early admission to PICU for CPAP would a) decrease the need for endotracheal intubation for invasive mechanical ventilation and b) improve the outcomes of acute respiratory failure in infants and children with impaired immunity. 


\section{Methods}

We undertook an open, parallel-group RCT between January 2013 to January 2016 in three children's hospitals with large bone marrow transplant programmes in the UK. Research ethics committee approval was obtained from the National Research Ethics Service Committee London Riverside (reference 12/LO/1051). The protocol was registered prior to trial opening (ISRCTN82853500) and is available as an electronic supplement. The study was funded by Great Ormond Street Hospital Children's Charity (Registered Charity No. 235825).

\section{Participants}

Children aged less than 18 years with impaired immunity (as a result of a primary diagnosis, therapy or a combination of both - see full protocol in electronic supplement) expected to last for at least three months and who developed acute (or acute on chronic) respiratory failure were eligible for the study.

Importantly, the standard of care in the participating units (and in UK healthcare system in general) is to admit to intensive care only when organ support is thought to be necessary. Children are not typically admitted for 'observation.'

Acute respiratory failure was defined by:

1) new onset of hypoxaemia: $\mathrm{SpO}_{2}<90 \%$ in air.;

2) tachypneoa: greater than $90^{\text {th }}$ centile for age unless receiving an opiate infusion (respiratory rate $55<12$ months, 4512 months to $<2$ years, $30 \geq 2$ years to $<4$ years, $25 \geq 4$ years to $<10$ years and $20 \geq 10$ years to $<18$ years); and

3) new or increased pulmonary infiltrates on chest x-ray developing within 48 hours.

Acute on chronic respiratory failure was defined by:

1) an increase of more than $20 \%$ above baseline supplemental oxygen;

2) an increased respiratory rate; and

3) new or increased pulmonary infiltrates on chest x-ray.

The cause of acute respiratory failure was not relevant to eligibility for the study. Recruitment within 24 hours of meeting all three criteria was required. Potential study participants were identified by hospital ward staff in collaboration with intensive care outreach teams who alerted the research study teams.

Exclusion criteria were: ongoing receipt of invasive mechanical ventilation for non-respiratory indications; emergency PICU admission for invasive mechanical ventilation for other conditions independent of the degree of acute respiratory failure e.g. for shock, reduced level of 
consciousness, or seizures, as deemed by the PICU team; recent oesophageal/gastric surgery; end-of-life care plan in place with limitation of resuscitation; life expectancy less than 12 months; and already receiving care on the PICU.Formal written consent was obtained from parents prior to inclusion in the study.

\section{Randomisation}

Participants were allocated 1:1 to early CPAP (intervention group) or standard care (control group) using a secure, web-based, randomisation system (www.sealedenvelope.com). Minimisation was performed on age ( $<12$ months/ $\geq 12$ months) and on bone marrow transplantation status (yes/no). Each participant was allocated with $80 \%$ probability to the group that minimised between group differences in these factors among all participants recruited to the trial to date and to the alternative group with $20 \%$ probability.

\section{Procedures}

Four Levels of respiratory support were defined (Figure 2a: Level 1 - inspired oxygen therapy on the ward; Level 2 - CPAP at 6-10 $\mathrm{cm} \mathrm{H} \mathrm{H}_{2} \mathrm{O}$; Level 3 - non-invasive bilevel positive airway pressure (BiPAP); and Level 4 - endotracheal intubation and mechanical ventilation). High flow nasal cannula oxygen (HFNC) was not permitted in the context of this study.

Participants allocated to the intervention group were admitted to the PICU to receive CPAP (Level 2 respiratory support) of at least $6 \mathrm{~cm} \mathrm{H}_{2} \mathrm{O}$ with supplemental oxygen for a minimum of 12 hours per day for four consecutive days. The interface for delivering CPAP and all other care was determined by the primary PICU clinical team.

Participants allocated to the standard care group remained on the ward and received supplemental oxygen (Level 1 respiratory support) to maintain oxygen saturation in accordance with standard local practice. The choice of oxygen delivery system and dose were determined by the primary ward clinical team as were all other aspects of care

Participants remained on CPAP or supplemental oxygen unless they either met pre-specified criteria for escalation or developed another condition (e.g. shock or encephalopathy) that required endotracheal intubation and invasive mechanical ventilation.

Briefly, criteria for escalation were (see full protocol in electronic supplement): respiratory acidosis (defined as an increase in $\mathrm{PCO}_{2}$ accompanied by a $\mathrm{pH} \leq 7.20$ for more than one hour); persistent moderate hypoxaemia (defined as either a $\mathrm{PaO}_{2}: \mathrm{FiO}_{2}<85 \mathrm{mmHg}\left(11.3 \mathrm{kPa}\right.$ ) or $\mathrm{SpO}_{2}: \mathrm{FiO}_{2}<98$ $\mathrm{mmHg}$ for more than one hour or $\mathrm{SpO}_{2}<90 \%$ for more than one hour despite optimised therapy); severe hypoxaemia (defined as failure to maintain $\mathrm{SpO}_{2}>80 \%$ at any time); evidence of persistent 
inadequate oxygen delivery (defined as an increase in serum lactate or $\mathrm{ScvO}_{2}$ persistently $<65 \%$ ).

While the criteria to escalate support or to go directly to endotracheal intubation and invasive mechanical ventilation were protocolised, decisions to stepdown respiratory support were at the discretion of the clinical team (aside from a step-down from Level 2 to Level 1 for the intervention group which was not permitted before the end of the four-day intervention period). "The choice of respiratory support settings within each of the four Levels (for example the choice of CPAP or BIPAP settings) was at the discretion of the clinical team."

\section{Outcomes}

The primary outcome was endotracheal intubation and invasive mechanical ventilation (Level 4 respiratory support) within 30 days post-randomisation.

Secondary outcomes were: within 30 days of randomisation; mortality, days free from any ventilatory support, maximum and aggregate Paediatric Logistic Organ Dysfunction score (PELOD), receipt of Level 2 or Level 3 respiratory support, and days free from supplemental oxygen (above pre-acute respiratory failure); and hospital, 90 day and one-year mortality.

\section{Sample size calculation}

Using UK Paediatric Intensive Care Audit Network (PICANet) data, 75\% of PICU admissions with impaired immunity and acute respiratory failure received endotracheal intubation for invasive mechanical ventilation. No accurate estimates for oxygen use in children with impaired immunity on the ward were available. From discussions with ward staff we estimated that approximately twice as many children received oxygen on the ward as were admitted to PICU. Therefore, we anticipated an endotracheal intubation rate of approximately $35-40 \%$ in the control group. Using a similar relative risk reduction ( $60 \%)$ as reported in the adult RCTs, we anticipated a decrease in the endotracheal intubation rate from $35 \%$ to $14 \%$. A sample of 148 children (74 in each group) would be required to detect with $80 \%$ power and a type 1 error rate of $5 \%$ (two-sided).

\section{Statistical analysis}

Analyses were specified and lodged a priori according to a Statistical Analysis Plan (see electronic supplement). All analyses were conducted by intention-to-treat, with a two-sided $p$ value of $<0.05$ taken to indicate a statistically significant result. The primary effect estimate was the relative risk of endotracheal intubation for invasive mechanical ventilation at 30 days post-randomisation, with statistical significance assessed by Fisher's exact test.

As a secondary analysis, the primary outcome was also analysed by multivariable logistic 
regression adjusted for baseline covariates of age, chronic diagnosis, bone marrow transplantation, suspected cause of acute respiratory failure, weight for age $Z$ score (using the British 1990 growth reference) and Paediatric Index of Mortality 2 (2016 recalibration). The baseline covariates were selected a priori for their anticipated strong association with the outcome, no further selection of covariates was performed based on imbalance at baseline or significance in univariable analyses.

Secondary outcomes were analysed by logistic regression for binary outcomes (mortality, receipt of Level 2/3 respiratory support) or linear regression for continuous outcomes (PELOD, days free from ventilatory support/supplemental oxygen) adjusted for the same baseline covariates as above. Due to the small sample size, no subgroup analyses were planned. Requests for sharing of deidentified participant data will be considered by the investigators.

\section{Role of the funding source.}

The funder of the study had no role in study design, data collection, data analysis, data interpretation, or writing of the writing of the report. The corresponding author had full access to all the data in the study and had final responsibility for the decision to submit for publication. 


\section{Results}

Between $7^{\text {th }}$ January 2013 to $31^{\text {st }}$ January 2016,114 children who met inclusion criteria were identified on screening, 19 of these met one or more exclusion criteria and 42 were recruited to the study (Figure 1) and followed for one year. No participants were lost to follow-up. Baseline characteristics were similar in both groups (Table 1). Notably, 20 of $42(48 \%)$ participants were admitted following a bone marrow transplant.

Recruitment was approximately one third of anticipated resulting, in part, from an overestimation of oxygen use on the ward and relatively low levels of consent to participate. At review after 19 months the Trial Steering Committee made a decision, supported by the Data Monitoring Committee and funder, to continue recruitment to the end of the original, planned, three-year period but not to extend beyond this date. This decision was based on four factors: a lack of safety concerns; recognition of the incorrect assumptions contributing to the power calculation and recruitment targets; the value of any trial data in this high-risk population since none were available; and a recognition that extending the trial may be problematic because of evolving practice including the use of high flow nasal cannula (HFNC) therapy.

Two of the 21 participants in the early group were not admitted to PICU because of lack of bed availability (Table 2). Of the 19 admitted, 18 received CPAP - although only 11 completed CPAP for the prescribed minimum allocation of four days of at least 12 hours of CPAP. These protocol deviations were principally because of perceived discomfort with CPAP. HFNC oxygen was administered outside of the protocol to four participants in the intervention group and to three in the control group.

All cases $>10 \mathrm{~kg}$ received CPAP/BiPAP via full-face mask from either Evita $4 / \mathrm{XL}$, Servo $U$ ventilators, or the Philips Respironics BiPAP Vision. Selected infants received CPAP via nasal mask via Servo-U ventilators or nasal masks or prongs via EME infant flow drivers

\section{Primary outcome}

Endotracheal intubation for invasive mechanical ventilation within 30 days in the early CPAP group was double that for the standard care group $(10 / 21,47.6 \%$ versus $5 / 21,23.8 \%$, relative risk 2.00 , $95 \%$ confidence interval 0.82 to $4.86, p=0.11$; Table 3 , Figure 3 ). This was not altered by adjustment for the pre-specified baseline covariates (Table 3). 


\section{Secondary outcomes}

Mortality at 30 days, at 90 days and at hospital discharge was significantly higher in the early CPAP group while one-year mortality was similar for both groups (Table 3, Figure 4). These findings persisted following adjustment for covariates.

Receipt of Level 2 or Level 3 respiratory support and days alive and free from ventilatory support or supplemental oxygen were similar for both groups. Maximum and aggregate PELOD scores in the first 30 days trended towards being greater in the intervention group - and were significant following adjustment for covariates.

\section{Adverse events}

Adverse events were rare - one pneumothorax was reported in the intervention group and one haemothorax in the control group. 


\section{Discussion}

SCARF is the only RCT performed to date in children with the high-risk combination of impaired immunity and acute respiratory failure. We found no evidence that a strategy of early PICU admission for CPAP was a superior approach to the management of acute respiratory failure in children with severely impaired immunity. Indeed, this strategy may have contributed to an increased early mortality. In addition to informing on a lack of benefit from early PICU admission for CPAP in this population, this study reinforces the overall high mortality for this population - $50 \%$ $(21 / 42)$ at one year.

This trial has a number of weaknesses. First, it is significantly underpowered for the primary outcome. A major contributor to this was the lack of data on ward level oxygen use in each institution (or in the literature), meaning that the assumptions underpinning the sample size calculation were incorrect. The introduction of electronic databases of vital sign observations means that this should be avoidable in the future. Second, our definition of respiratory failure was not a validated measure, but rather sought to identify the earliest point at which a reasonable body of paediatric intensivists might consider using CPAP. Third, adherence to the CPAP protocol was challenging either due to a lack of PICU bed availability or, predominantly, to intolerance of a facemask. The intention-to-treat analysis, however, likely provides a 'real-life' comparison of the clinical effectiveness of these two strategies (rather than a narrower efficacy study). Fourth, the choice of primary outcome measure could be criticised. Endotracheal intubation was chosen as it had been used in previous RCTs in this field,(4-6) and was anticipated to have a higher event rate than mortality while closely associated with it. The potential problem with endotracheal intubation rate is that both part of the intervention and the outcome are determined by the clinical team. Fifth with this sample size and the lack of a suitable validated severity-of-illness measure outside of the ICU we cannot exclude the CPAP group being more unwell at baseline. Finally, reflecting on the design of this RCT in 2012, high-flow nasal cannula oxygen (HFNC) was not assessed in the study as its use was not commonplace at this time. We are very aware of the importance of this question. We have recently completed a randomised controlled feasibility trial comparing high-flow nasal cannula oxygen.(7) However, Chalmers reminds us of the importance of reporting of all trial data.(8) Perhaps this is especially true when there are no other data for comparison.

This RCT, however, has the following strengths. First, it is a formal, multicentre RCT following CONSORT guidance with a pre-registered protocol and statistical analysis plan. Such studies are relatively rare in critically-ill children. Second, the results are consistent across all outcome measures with standard care either superior to early CPAP or trending in that direction. Third, the Fragility Index for SCARF is 10(9) - meaning all 10 patients who received endotracheal intubation 
in the early CPAP group would have had to avoid this for the study to suggest superiority of CPAP as a recommended approach.

Recent work in adults has similarly failed to show an advantage for non-invasive ventilation. The IVNIctus trial which recruited around 350 adults with haematological or solid cancers suggested equivalence for early NIV with oxygen therapy alone.(10) The Efraim multinational prospective cohort study of immunocompromised adults with acute hypoxaemic respiratory failure also found no reduction in intubation or mortality with NIV. Interestingly HFNC was associated with a reduction in need for intubation but not mortality.(11) NIV failure is consistently associated with poor outcome. (12),(13) Non-invasive ventilation is being discouraged in this population.(14) To this end, our data are not surprising in this context.

The important unanswered question is 'why was early CPAP not superior?' In answering this, both the impact of CPAP use and early admission to PICU have to be considered as both were part of the intervention. Might CPAP have had a direct deleterious effect? This seems unlikely given the data supporting positive end expiratory pressure.(15) However, overdistension can certainly be harmful in healthy lungs or focal ARDS or some cases of severe lung injury with non-recruitable lung. Here excessive PEEP can lead to overdistention of recruitable or healthy lungs elements. $(16,17)$ While perhaps unlikely to be the full explanation at the low levels of non-invasive CPAP provided in in this trial, the timing may be crucial. Our protocol prompted the use of CPAP significantly earlier in an episode of respiratory failure in the intervention group. Therefore, these relatively healthy lungs may be vulnerable to overdistension. As with all treatments the potential for benefit or harm is influenced by the baseline risk of the population to which it is applied. CPAP may indeed be a highly effective treatment for respiratory failure in children with impaired immunity - but our data do not support its use at this very early stage.

Perhaps the setting is the important difference. PICU involves significantly more intensive monitoring with higher staffing levels. It may be that earlier PICU admission results in a lower tolerance of physiological instability and greater levels of clinical intervention which come with additional risks in a patient who is relatively well having been admitted earlier than is standard practice. This might include higher concentrations of supplemental oxygen being administered(18) or an increased frequency of intervention with risks for nosocomial infection (e.g. sedative infusions, new venous access) especially in this immunocompromised population. A third arm which analysed CPAP administration on the ward would have been valuable in understanding this. The role of HFNC oxygen, which can be delivered on the ward more easily than CPAP, deserves consideration in this context. Of course, in healthcare systems with a lower threshold for PICU admission that would include our control group entering PICU, the risks and benefit of CPAP are likely to differ. 
Recent improvements in critical care have included refinements in our understanding of which patients benefit most from common interventions. Perhaps our findings concerning early CPAP can be best understood in this context.(19)

\section{Conclusion}

This RCT found no advantage for early PICU admission for CPAP in children with acute (or acute on chronic) respiratory failure in the setting of impaired immunity. There was increased early mortality associated with early PICU admission for CPAP. However, this effect was not apparent by one year of follow-up.

\section{Acknowledgements}

The authors would like to thank the medical and nursing staff at each trial site, the Paediatric Intensive Care Society Study Group (PICS-SG) and the patients and their families who contributed to this study. We also are grateful to Rahi Jahan at ICNARC and members of the Trial Steering Committee and Data Monitoring Committee for their assistance in performing this study. 


\section{References}

1. López-Fernández $\mathrm{Y}$, Azagra AM-D, la Oliva de $\mathrm{P}$, et al.: Pediatric Acute Lung Injury Epidemiology and Natural History study: Incidence and outcome of the acute respiratory distress syndrome in children. Crit Care Med 2012; 40:3238-3245

2. Peters MJ, Agbeko RS: Optimism and no longer foolishness? Haematology/oncology and the PICU. Intensive Care Med 2014; 40:1589-1591

3. Duncan CN, Lehmann LE, Cheifetz IM, et al.: Clinical Outcomes of Children Receiving Intensive Cardiopulmonary Support During Hematopoietic Stem Cell Transplant*. Pediatr Crit Care Med 2013; 14:261-267

4. Hilbert G, Gruson D, Vargas F, et al.: Noninvasive ventilation in immunosuppressed patients with pulmonary infiltrates, fever, and acute respiratory failure. $N$ Engl $J$ Med 2001; 344:481-487

5. Squadrone V, Massaia M, Bruno B, et al.: Early CPAP prevents evolution of acute lung injury in patients with hematologic malignancy. Intensive Care Med 2010; $36: 1666-1674$

6. Antonelli M: Noninvasive Ventilation for Treatment of Acute Respiratory Failure in Patients Undergoing Solid Organ Transplantation: A Randomized Trial. JAMA: The Journal of the American Medical Association 2000; 283:235-241

7. Ramnarayan $\mathrm{P}$, Lister $\mathrm{P}$, Dominguez $\mathrm{T}$, et al.: FIRST-line support for Assistance in Breathing in Children (FIRST-ABC): protocol for a multicentre randomised feasibility trial of non-invasive respiratory support in critically ill children. BMJ Open 2017; $7: \mathrm{e} 016181-10$

8. Chalmers I, Glasziou P, Godlee F: All trials must be registered and the results published. BMJ 2013; 346:f105

9. Walsh M, Srinathan SK, McAuley DF, et al.: The statistical significance of randomized controlled trial results is frequently fragile: a case for a Fragility Index. Journal of Clinical Epidemiology 2014; 67:622-628

10. Lemiale V, Mokart D, Resche-Rigon M, et al.: Effect of Noninvasive Ventilation vs Oxygen Therapy on Mortality Among Immunocompromised Patients With Acute Respiratory Failure. JAMA 2015; 1

11. Azoulay E, Pickkers $P$, Soares $M$, et al:: Acute hypoxemic respiratory failure in immunocompromised patients: the Efraim multinational prospective cohort study. Intensive Care Med 2017; 316:1-12

12. Azoulay $\mathrm{E}$, Lemiale $\mathrm{V}$, Mokart $\mathrm{D}$, et al:: Acute respiratory distress syndrome in patients with malignancies. Intensive Care Med 2014; 40:1106-1114

13. Neuschwander A, Lemiale V, Darmon M, et al.: Noninvasive ventilation during acute respiratory distress syndrome in patients with cancer: Trends in use and outcome. Journal of Critical Care 2017; 38:295-299 
14. Neuschwander A, Lemiale V, Azoulay E: Noninvasive ventilation for ARDS in patients with cancer: Still possible or there continues to be a learning curve? In reply. Journal of Critical Care 2017; 40:274

15. Amato MBP, Meade MO, Slutsky AS, et al.: Driving Pressure and Survival in the Acute Respiratory Distress Syndrome. N Engl J Med 2015; 372:747-755

16. Bersten AD, Kavanagh BP: A metabolic window into acute respiratory distress syndrome: stretch, the "baby" lung, and atelectrauma. American Journal of Respiratory and Critical Care Medicine 2011; 183:1120-1122

17. Puybasset L, Gusman P, Muller JC, et al.: Regional distribution of gas and tissue in acute respiratory distress syndrome. III. Consequences for the effects of positive end-expiratory pressure. CT Scan ARDS Study Group. Adult Respiratory Distress Syndrome. Intensive Care Med 2000; 26:1215-1227

18. Ray S, Rogers L, Raman S, et al.: Liberal oxygenation in paediatric intensive care: retrospective analysis of high-resolution SpO2 data. Intensive Care Med 2016; 43:146-147

19. Kox M, Pickkers P: "Less is more" in critically ill patients: not too intensive. JAMA Intern Med 2013; 173:1369-1372 


\section{Figures}

Figure 1 Flow of participants through RCT

(HFNC=high flow nasal cannulae)

Figure 2 Summary of Randomised Trial design Maximum Level of Support Received by Participants in Each Arm

A) Four Levels of respiratory support were described. Decision to escalate between Levels was protocolised. Settings within each Level were at the discretion of clinical staff as were decisions to de-escalate to a lower Level (other than de-escalation from CPAP in the intervention group which was required to be delivered for four days for a minimum of 12 hours).

B) The Maximum level of respiratory support received by participants in each group. Intervention group: 5 cases progressed through all levels of respiratory support and 5 were intubated for acute indications (shock, seizure, etc..) ${ }^{a}$ in two cases no ICU bed was available within 24 hours and cases remained on the ward. Control group: 2 were intubated following stepping-up through all four levels of respiratory support and 3 were intubated for acute indications.

Figure 3 Cumulative incidence of Level 4 respiratory support by treatment group to 30 days from randomisation

Figure 4 Kaplan-Meier plots of time to death, by treatment group, to (A) 90-days and (B) one year from randomisation

\section{Tables}

\section{Table 1 Comparison of baseline characteristics by treatment group}

CPAP, continuous positive airways pressure; IQR, interquartile range; PIM2r (2016), Paediatric Index of Mortality 2, 2016 recalibration; SD, standard deviation PELOD Pediatric logistic organ dysfunction score 
Table 2 Comparison of clinical management by treatment group

CPAP, continuous positive airways pressure; IQR, interquartile range; PICU, paediatric intensive care unit; $\mathrm{SD}$, standard deviation HFNC, High flow nasal cannula.

Table 3 Comparison of outcomes by treatment group

Table S1 Summary of sedatives administered during intervention period (Days 1-4) 
Figure 1. Flow of participants through RCT 
Figure 2 Summary of Randomised Trial design and the maximum level of support received by participants in each arm. 
Figure 3. Cumulative incidence of Level 4 respiratory support by treatment group to 30 days after randomisation 
Figure 4. Kaplan-Meier plots of time to death by treatment group to (A) 90 days and (B) 1 year after randomisation 
Table 1. Comparison of baseline characteristics by treatment group

a Factor included in minimisation algorithm; b One patient in the standard care group was missing weight;

c Weight for age $Z$ score calculated using British 1990 growth reference; d Patients may have more than one cause of impaired immunity; ${ }^{~}$ One patient in the standard care group was missing supplemental $\mathrm{O} 2$ requirement $-{ }^{f} P E L O D$ - note these score includes the 24 hours post-randomisation, so are a combination of baseline and early post-randomization care. 
Table 2. Comparison of clinical management by treatment group

a One patient in the standard care group was missing length of stay in PICU

b In two cases a PICU bed was not available in 24 hours, and in 1 CPAP was not tolerated

C Includes 8 cases who did not complete the full intervention of CPAP for $>12$ hours / day for 4 days

d intubated without stepping up through levels (1)-2-3 respiratory support 
Table 3. Comparison of outcomes by treatment group

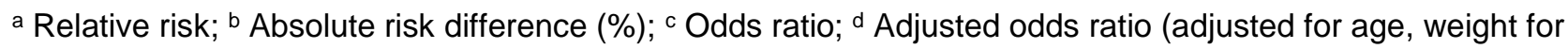
age $Z$ score, bone marrow transplant and PIM2r (2016) score); ${ }^{e}$ Mean difference; ${ }^{\dagger}$ Adjusted mean difference (adjusted for age, weight for age Z score, bone marrow transplant and PIM2r (2016) score)

CPAP, continuous positive airways pressure; PELOD, Paediatric Logistic Organ Dysfunction; PIM2r (2016), Paediatric Index of Mortality 2, 2016 recalibration; SD, standard deviation 